\title{
Unser Weg zu Ihren Zielen: Strategie 2017-2020
}

\author{
Jürg Schlup \\ Dr. med., Präsident der FMH
}

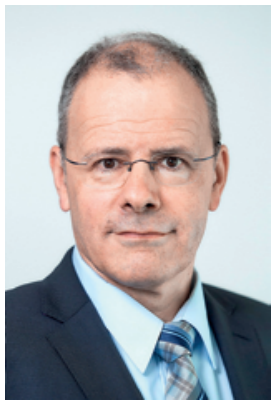

Wer gute Vorsätze für das nächste Jahr fasst, wird mit etwas Glück am Ende des Jahres zu den etwa 20 Prozent der Menschen gehören, die sich noch an ihre Vorsätze erinnern und sie länger als drei Monate durchgehalten haben. Ein Dachverband von über 70 Ärzteorganisationen darf sich aber nicht auf Vorsätze und Glück verlassen: Weil wir Ziele im Interesse unserer 40000 Mitglieder nur durch die Zusammenarbeit vieler beteiligter Personen und Akteure erreichen können, ist eine gut fundierte Strategie - und damit die Festlegung von Haupt- und Subzielen, Massnahmen, Verantwortlichkeiten und Controlling-Prozessen - für uns unabdingbar.

Entsprechend haben wir im FMH-Zentralvorstand eine Strategie für die jüngst begonnene Legislatur erarbeitet. Um sicherzustellen, dass die darin festgelegten Ziele unsere wichtigsten Herausforderungen wider-

\section{Einige der anstehenden Aufgaben sind nur mit Ihrer Unterstützung lösbar.}

spiegeln, befragten wir Ärztekammer-Delegierte, gesundheitspolitische Experten und die Kadermitglieder des Generalsekretariats, wo sie die für die FMH wichtigsten Herausforderungen sehen. Auf der Grundlage dieser Befragungsergebnisse formulierte der Zentralvorstand in drei Workshops Ziele und konkretisierte dazugehörige Massnahmen. Genaueres hierzu erfahren Sie in einem Artikel dieser Ausgabe auf Seite 4.

Die nun in der Strategie verankerten Herausforderungen, die von der Ärzteschaft und unserem politischen Umfeld formuliert wurden, verstehen wir als Aufgaben, für deren Erfüllung wir uns entschlossen einsetzen. Einige dieser Aufgaben sind jedoch nur mit Ihrer Unterstützung lösbar. Dies gilt vor allem für die gemäss unseren Befragungen unangefochtene Top-Priorität Tarifrevision und Erhalt der Tarifautonomie, aber auch für die angestrebte Stärkung des Zusammenhalts des Verbandes und einen geschlossenen Auftritt nach aussen.

Diese Prioritäten lassen aufmerken, wenn man an die teils heftigen Tarifdiskussionen denkt, und daran, dass unser Tarifstruktur-Vorschlag im Juni 2016 mit 54\%

abgelehnt wurde, obwohl bekannt war, dass wir innerhalb der engen Zeitvorgaben des Bundes keinen besseren und breiter abgestützten Tarifvorschlag vorlegen können würden. Dies hat unsere politische Situation nicht einfacher gemacht: Ohne Tarifvorschlag stehen wir mit leeren Händen da, sogar liberale Parlamentarier fordern mittlerweile einen Staatstarif. Zwar arbeiten wir unter Hochdruck an Nachbesserungen des Tarifs, sind aber auf ein Entgegenkommen der Politik angewiesen: denn ein nachgebesserter, unter den Ärzteorganisationen demokratisch abgestützter und mit den Tarifpartnern abgestimmter Revisionsvorschlag kann nicht vor 2018 finalisiert werden. Und dieser gemeinsame Weg ist für uns nach wie vor der einzig richtige - auch wenn er Zeit braucht.

Dass die Ärzteschaft in dieser Situation der Tarifautonomie und -revision sowie dem Überwinden interner Differenzen eine hohe Bedeutung beimisst, ist eine wichtige Vorbedingung für einen Erfolg - entscheidend wird aber sein, was jede Fachdisziplin bereit ist, dafür zu geben! Schliesst der Ruf nach Einigkeit auch ein, selber nicht gleich auszuscheren, wenn man Abstriche machen muss? Ist der Wunsch nach $\mathrm{Zu}$ sammenhalt stark genug, um auf öffentliche Schlagabtausche zu verzichten? Meint die Forderung nach Solidarität nicht nur die Solidarität der Anderen, sondern auch die eigene Bereitschaft dazu? Dann hätten wir eine gute Chance, denn: Tarifautonomie und Zusammenhalt erfordern die Bereitschaft aller Ärzteorganisationen und Tarifpartner, für eine Lösung zu arbeiten und einzustehen, die für niemanden perfekt sein wird - und es dennoch wert ist.

Es braucht die Bereitschaft aller Beteiligten, für eine Lösung zu arbeiten, die nicht perfekt sein wird - und es dennoch wert ist.

Mit unserer Arbeit auf Basis der neuen Strategie geben wir unser Bestes für unsere Mitglieder. Am Ende der Legislatur werden wir alle unsere Ziele noch kennen und deutlich länger als drei Monate dafür gearbeitet haben. Ob wir sie erreicht haben werden, hängt nicht zuletzt auch von Ihnen ab - wir zählen auf Sie! 